\title{
Curcumin suppresses transforming growth factor- $\beta 1$-induced cardiac fibroblast differentiation via inhibition of Smad-2 and p38 MAPK signaling pathways
}

\author{
HUZI LIU ${ }^{1 *}$, AIJUN LIU², CHUNLI SHI ${ }^{3}$ and BAO LI ${ }^{4}$ \\ ${ }^{1}$ Department of Cardiac Surgery, Shanxi Cardiovascular Hospital, Taiyuan, Shanxi 030024; ${ }^{2}$ Pediatric Heart Center, \\ Beijing Anzhen Hospital, Capital Medical University, Beijing 100029; ${ }^{3}$ Outpatient Department and \\ ${ }^{4}$ Department of Cardiology, Shanxi Cardiovascular Hospital, Taiyuan, Shanxi 030024, P.R. China
}

Received November 16, 2014; Accepted December 10, 2015

DOI: $10.3892 /$ etm.2016.2969

\begin{abstract}
The differentiation of cardiac fibroblasts (CFs) into myofibroblasts and the subsequent deposition of the extracellular matrix is associated with myocardial fibrosis following various types of myocardial injury. In the present study, the effect of curcumin, which is a pharmacologically-safe natural compound from the Curcuma longa herb, on transforming growth factor (TGF)- $\beta 1$-induced CFs was investigated, and the underlying molecular mechanisms were examined. The expression levels of $\alpha$-smooth muscle actin (SMA) stress fibers were investigated using western blotting and immunofluorescence in cultured neonatal rat CFs. Protein and mRNA expression levels of $\alpha$-SMA and collagen type I (ColI) were determined by western blotting and reverse transcription-quantitative polymerase chain reaction. In addition, the activation of Smad2 and p38 was examined using western blotting. Curcumin, SB431542 (a TGF- $\beta$ R-Smad2 inhibitor) and SB203580 (a p38 inhibitor) were used to inhibit the stimulation by TGF- $\beta 1$. The results demonstrated that the TGF- $\beta 1$-induced expression of $\alpha$-SMA and ColI was suppressed by curcumin at the mRNA and protein levels, while SB431542 and SB203580 induced similar effects. Furthermore, phosphorylated Smad-2 and p38 were upregulated in TGF- $\beta 1$-induced CFs, and these effects were substantially inhibited by curcumin administration. In conclusion, the results of the present study demonstrated that treatment with curcumin effectively
\end{abstract}

Correspondence to: $\mathrm{Mr}$. Bao Li, Department of Cardiology, Shanxi Cardiovascular Hospital, 18 Yifen Street, Taiyuan, Shanxi 030024, P.R. China

E-mail: libao_sch@163.com

*Contributed equally

Abbreviations: CFs, cardiac fibroblasts; $\alpha$-SMA, $\alpha$-smooth muscle actin; ColI, collagen type I

Key words: curcumin, cardiac fibroblast, transforming growth factor- $\beta 1$, Smad2, p38 mitogen-activated protein kinase suppresses TGF- $\beta 1$-induced CF differentiation via Smad-2 and p38 signaling pathways. Thus, curcumin may be a potential therapeutic agent for the treatment of cardiac fibrosis.

\section{Introduction}

Cardiac fibroblast differentiation, excessive biosynthesis and destruction of the interstitial extracellular matrix (ECM) in the ventricles of the heart are key features of cardiac fibrosis, which is a consequence of cardiac remodeling initiated by pathological events associated with various cardiovascular disorders (1). Induced by transforming growth factor (TGF)- $\beta 1$ and other factors, cardiac fibroblasts (CFs) differentiate into $\alpha$-smooth muscle actin (SMA) fiber-rich cardiac myofibroblasts that facilitate contractility and increase ECM modulation ability (1). Although these changes are important for wound repair and are beneficial for the maintenance of cardiac function, continuous myocardial fibrosis may result in abnormal myocardial stiffness and, ultimately, ventricular dysfunction (1).

Curcumin, 1,7-bis(4-hydroxy-3-methoxyphenyl)-1, 6-heptadiene-3,5-dione, is a natural polyphenolic compound from the Curcuma longa herb, possessing multiple biological and medicinal activities (2). The pharmacological safety of curcumin has previously been demonstrated in various animal models, and curcumin is nontoxic even at high doses (3). Previous studies have demonstrated that curcumin has antioxida, anti-inflammatory, anti-proliferative, pro-apoptotic and anti-carcinogenic properties (4-8). Furthermore, previous investigations into the effects of curcumin in heart disease have indicated that curcumin has a regulatory role in the cardiac remodeling process; curcumin has been demonstrated to ameliorate and reverse cardiac fibrosis, cardiac hypertrophy and heart failure in animal models (9-13). Therefore, curcumin may represent a novel therapeutic strategy for the treatment of cardiac remodeling.

TGF- $\beta 1$ is a key mediator of the differentiation of fibroblasts to myofibroblasts (14), and this TGF- $\beta 1$-induced effect has been demonstrated to be associated with the Smad2 and p38 mitogen-activated protein kinase (MAPK) signaling pathways (15-17). Although it has previously been demonstrated 
that curcumin has an inhibitory effect on ECM secretion in cultured CFs (10), the effect of curcumin on the differentiation of CFs and the underlying mechanisms are yet to be fully elucidated. In the present study, using cultured CFs from neonatal rats, it was demonstrated that curcumin has an inhibitory effect on TGF- $\beta 1$-induced cardiac fibroblast differentiation. Furthermore, the role of Smad2 and p38 MAPK signaling in the activation of CFs and the anti-fibrotic mechanism of curcumin in the modulation of the TGF- $\beta 1$ induced effects was addressed.

\section{Materials and methods}

Reagents. TGF- $\beta 1$ was purchased from R\&D Systems, Inc. (Minneapolis, MN, USA). Curcumin and rabbit monoclonal anti- $\alpha$-SMA antibody (SP171) were purchased from Sigma-Aldrich (St. Louis, MO, USA). Rabbit polyclonal anti-collagen type I (ColI; ab34710) and -von Willebrand factor (ab6994) primary antibodies were purchased from Abcam (Cambridge, MA, USA), whereas mouse monoclonal anti-vimentin antibody (zm-0260), -glyceraldehyde-3-phosphate dehydrogenase (GAPDH) antibody (TA-08), and horseradish peroxidase (HRP)-conjugated goat anti-rabbit and goat anti-mouse immunoglobulin $(\mathrm{Ig}) \mathrm{G}$ secondary antibodies (ZB-2301 and ZB-2305) were purchased fromZhongsan Jinqiao Biotechnology Co., Ltd. (Beijing, China). Phosphorylated and non-phosphorylated monoclonal rabbit anti-rat Smad2 (\#3108 and \#5339) and p38 (\#9215 and \#2371) primary antibodies were purchased from Cell Signaling Technology, Inc. (Danvers, MA, USA). SB431542, a TGF- $\beta$ R-Smad2/3 inhibitor was purchased from Cayman Chemical Company (Ann Arbor, MI, USA) and SB20358, a p38 inhibitor, was purchased from Merck Millipore (Darmstadt, Germany). Cell culture materials and TRIzol ${ }^{\circledR}$ reagent were purchased from Thermo Fisher Scientific, Inc. (Waltham, MA, USA). The DNase and primers used for the reverse transcription-quantitative polymerase chain reaction (RT-qPCR) were purchased from Sunbiotech (Beijing, China).

Cell culture and treatment. The present study was conducted with the approval of the Ethics Committee of Experimental Animal Center of Shanxi Cardiovascular Hospital (Taiyuan, China), according to the regulations outlined by the National Institutes of Health Guidelines on the Use of Laboratory Animals. CFs were isolated from neonatal (1-3-days-old) Sprague-Dawley rats via trypsin digestion methods, as previously described (18). CFs were cultured in Dulbecco's Modified Eagle's Medium supplemented with 10\% fetal bovine serum, and were passaged using trypsin (1:3). Second passage CFs were used in the present study and were serum-starved for $24 \mathrm{~h}$ at $80 \%$ confluence in order to induce quiescence. Immunocytochemical analysis demonstrated that the purity of the CFs used was $>95 \%$, according to positive staining for vimentin and negative staining for von Willebrand factor.

In order to elucidate the potential effects of curcumin on the various signaling pathways in TGF- $\beta 1$-induced CFs, CFs were pretreated with $20 \mu \mathrm{mol} / 1$ curcumin, $10 \mu \mathrm{M}$ TGF- $\beta \mathrm{R}-\mathrm{Smad} 2$ inhibitor (SB431542) or $10 \mu \mathrm{M}$ p38 MAPK inhibitor (SB203580) for $30 \mathrm{~min}$, prior to treatment with recombinant
$10 \mathrm{ng} / \mathrm{ml} \mathrm{TGF}-\beta 1$ for $24 \mathrm{~h}$. In order to investigate the activation of Smad 2 and p38, CFs were treated with $10 \mathrm{ng} / \mathrm{ml}$ TGF- $\beta 1$ for $1 \mathrm{~h}$. Cells were subsequently harvested and stored at $-80^{\circ} \mathrm{C}$ prior to the determination of protein and mRNA expression levels.

Immunofluorescent staining. CFs were cultured on coverslips in 6 -well plates $\left(2.5 \times 10^{5}\right.$ cells/well). Growth was arrested and CFs were treated as described earlier. Following a 24-h treatment, CFs were fixed with $4 \%$ paraformaldehyde and permeabilized with $0.1 \%$ Triton X-100 (Applygen Technologies, Inc., Beijing, China). Non-specific binding was blocked via incubation with $10 \%$ normal goat serum (Applygen Technologies, Inc.). Subsequently, $\alpha$-SMA was detected using a Cy5-conjugated goat anti-rabbit $\alpha$-SMA polyclonal antibody (Abcam; ab6564) and stained cells were visualized using the BX51 Fluorescence Microscope (Olympus Corporation, Tokyo, Japan).

Western blotting. CFs were harvested in lysis buffer (Applygen Technologies, Inc.) containing $20 \mathrm{mM}$ Tris, $150 \mathrm{mM} \mathrm{NaCl}$, 1 mM EDTA, 1 mM EGTA, 1\% Triton X-100, 2.5 mM sodium pyrophosphate, $1 \mathrm{mM} \beta$-glycerol phosphate, $1 \mathrm{mM} \mathrm{Na} \mathrm{VO}_{4}$ (pH 7.5), $1 \mathrm{mM}$ phenylmethanesulfonyl fluoride, $1 \mathrm{mM}$ benzamidine, $10 \mu \mathrm{g} / \mathrm{ml}$ leupeptin and $10 \mu \mathrm{g} / \mathrm{ml}$ aprotinin. Protein concentrations were determined using a bicinchoninic acid protein assay kit (Pierce Biotechnology, Inc., Rockford, IL, USA), according to the manufacturer's protocol. Following boiling for $1 \mathrm{~min}$ to denature, $20 \mu \mathrm{g}$ protein was separated by $10 \%$ sodium dodecyl sulfate-polyacrylamide gel electrophoresis and transferred to polyvinylidene difluoride membranes (Bio-Rad Laboratories, Inc., Hercules, CA, USA). Membranes were blocked with $5 \%$ fat-free milk in Tris-buffered saline with Tween-20 (TBST; $10 \mathrm{mM}$ Tris $\mathrm{HCl}, 150 \mathrm{mM} \mathrm{NaCl}$ and $0.1 \%$ Tween-20) for $1 \mathrm{~h}$ at room temperature, and incubated overnight with primary antibodies $(1: 2,500)$ at $4^{\circ} \mathrm{C}$. Following each incubation, membranes were washed three times for 10 min with TBST and subsequently incubated with HRP-conjugated secondary antibody $(1: 5,000)$ for $1 \mathrm{~h}$ at room temperature. Using chemiluminescence (Applygen Technologies, Inc.), the membranes were scanned and quantified using Quantity-One software, version 4.2 (Bio-Rad Laboratories, Inc.), and the results were presented as the optical density of phosphorylated-protein/total protein or of the target protein/GAPDH.

$R N A$ isolation and RT-qPCR. Total RNA was extracted from CFs using TRIzol ${ }^{\circledR}$ reagent and treated with DNase, according to the manufacturer's protocols. RT-qPCR analyses were performed using an ABI 7300 system (Applied Biosystems; Thermo Fisher Scientific, Inc.). For each sample, $1 \mu \mathrm{g}$ RNA was utilized to synthesize cDNA using the Reverse Transcriptase kit (Promega Corporation, Madison, WI, USA), according to the manufacturer's protocol. The specific primer sequences used were as follows: $\alpha$-SMA forward, 5'-CATCAGGAA CCTCGAGAAGC-3', and reverse, 5'-TCGGATACTTCA GGGTCAGG-3'; ColI forward, 5'-CATAAAGGGTCATCG TGGCTTC-3', and reverse, 5'-GTGATAGGTGATGTTCTG GGAG-3'; and GAPDH forward, 5'-AACCTGCCAAGT ATG ATGACATCA, and reverse, 5'-TTCCACTGATATCCCAGC 
A
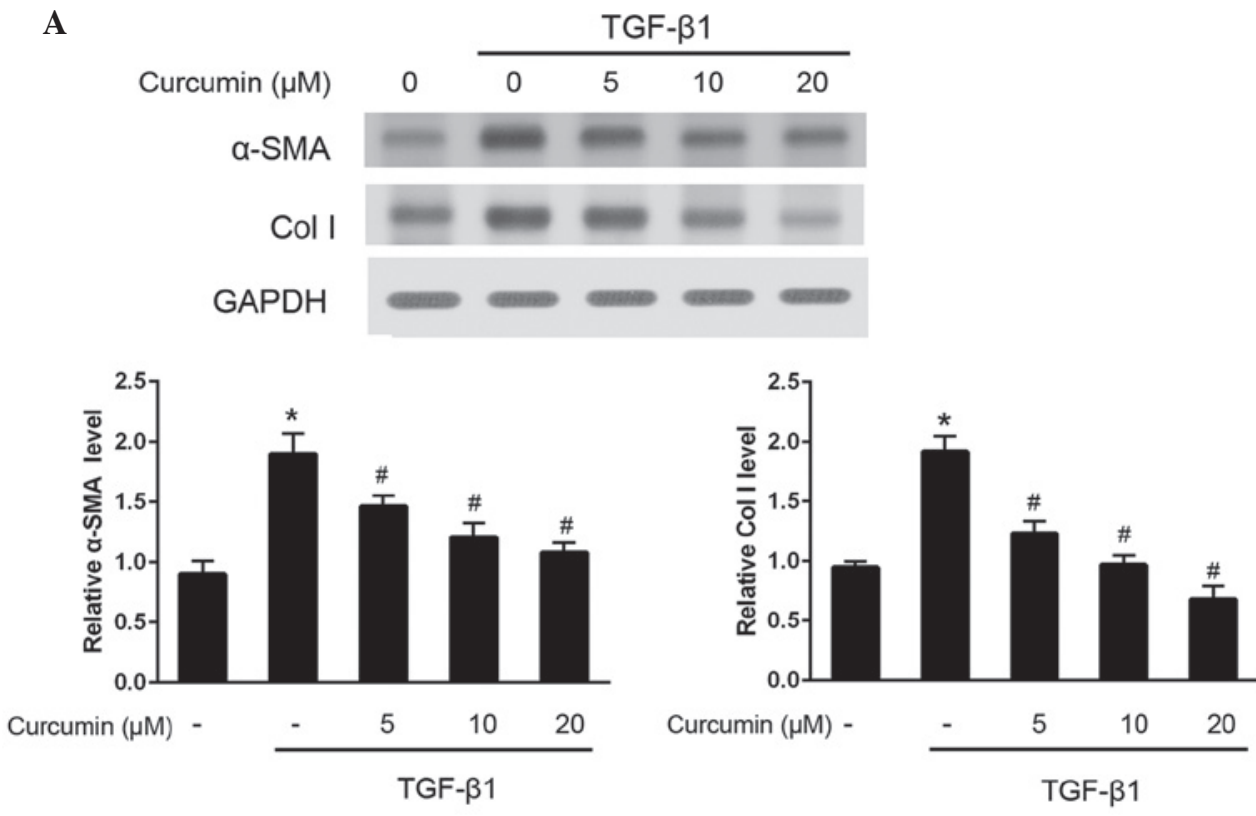

B

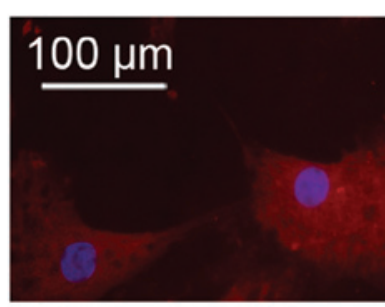

Control

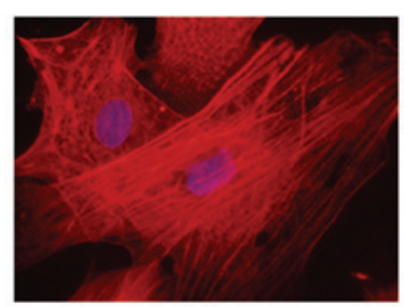

TGF- $\beta 1$

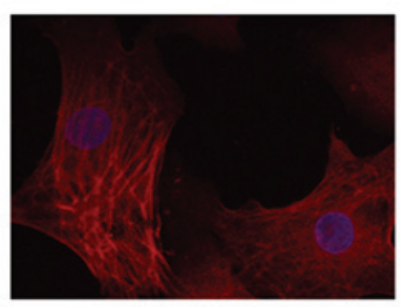

TGF- $\beta 1+$ curcumin

Figure 1. Curcumin suppressed $\alpha$-smooth muscle actin (SMA) and collagen I (ColI) protein expression levels. (A) Western blotting and corresponding densitometric quantification of $\alpha$-SMA, ColI and internal reference GAPDH expression in cardiac fibroblasts (CFs) treated with $10 \mathrm{ng} / \mathrm{ml}$ transforming growth factor (TGF)- $\beta 1$ in the presence of curcumin at various concentrations $(0,5,10$ and $20 \mu \mathrm{mol} / \mathrm{l})$ for $24 \mathrm{~h}$. (B) Immunofluorescence staining of $\alpha$-SMA in CFs following treatment with $20 \mu \mathrm{mol} / 1$ curcumin. $\alpha$-SMA and nuclei were stained with Cy5 (red) and DAPI (blue), respectively (bar, $100 \mu \mathrm{m}$ ). ${ }^{*} \mathrm{P}<0.05 \mathrm{vs}$. control group; ${ }^{\text { }} \mathrm{P}<0.05$ vs. TGF- $\beta 1$ group.
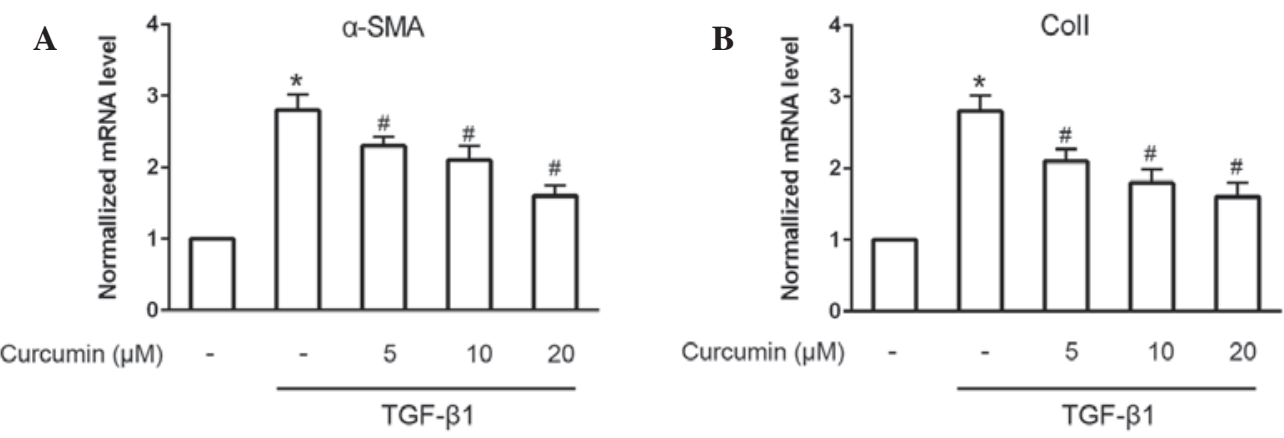

Figure 2. Reverse transcription-quantitative polymerase chain reaction analysis of (A) $\alpha$-smooth muscle actin (SMA) and (B) collagen I (ColI) mRNA expression levlels in cardiac fibroblasts (CFs) treated with or without 5,10 or $20 \mu \mathrm{mol} / 1$ curcumin for 30 min and incubated with $10 \mathrm{ng} / \mathrm{ml}$ transforming growth factor (TGF)- $\beta 1$ for $24 \mathrm{~h}$ was performed. Curcumin was found to suppress the $\alpha$-SMA and ColI mRNA expression levels. mRNA expression control CFs was normalized to 1 -fold, ${ }^{*} \mathrm{P}<0.05$ vs. control group; ${ }^{*} \mathrm{P}<0.05$ vs. TGF- $\beta 1$ group.

TGCT-3'. Target genes were amplified using SYBR Green PCR Master Mix (Applied Biosystems; Thermo Fisher Scientific, Inc.). The PCR cycling conditions were as follows: $95^{\circ} \mathrm{C}$ for $5 \mathrm{~min}$, followed by 40 cycles of $95^{\circ} \mathrm{C}$ for $35 \mathrm{sec}$ and $60^{\circ} \mathrm{C}$ for $1 \mathrm{~min}$, with a final extension step of $72^{\circ} \mathrm{C}$ for $5 \mathrm{~min}$. Relative mRNA levels were calculated using the $2^{-\Delta \Delta \mathrm{Cq}}$ method (19), where $\Delta \mathrm{Cq}$ was the difference between GAPDH and target gene critical threshold cycle $(\mathrm{Cq})$ values.
Statistical analyses. Statistical analyses in this study were conducted using the SPSS software, version 16.0 (SPSS, Inc., Chicago, IL, USA). The results are presented as the mean \pm standard error of the mean. Between-groups differences were assessed using one-way factorial analysis of variance. $\mathrm{P}<0.05$ was considered to indicate a statistically significant difference. Each assay in the present study was performed in triplicate. 
$\mathbf{A}$

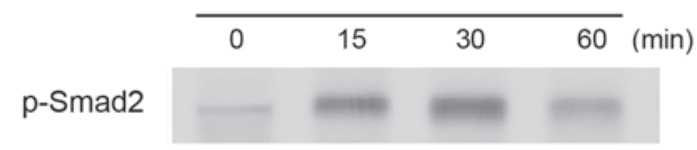

Smad2

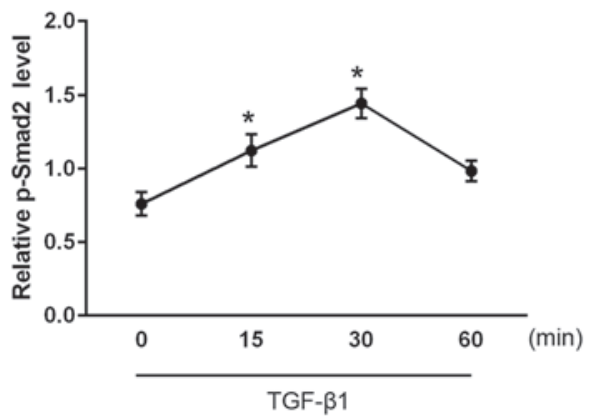

$\mathbf{B}$
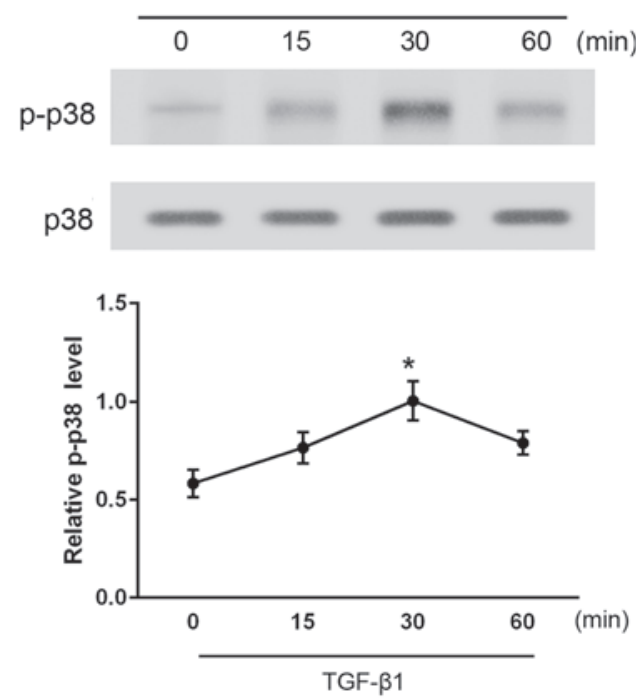

Figure 3. Effects of transforming growth factor (TGF)- $\beta 1$ on the phosphorylation of (A) Smad2 and (B) p38 over 60 min, as determined by western blotting. Total Smad2 and p38 was used as the control. Densitometric analysis was performed to quantify the phosphorylation levels, which are presented as the ratio between the optical densities of the p-Smad2 and total Smad2 and p-p38 and p38 bands, respectively. ${ }^{*} \mathrm{P}<0.05$ vs. control. p-, phosphorylated.

A

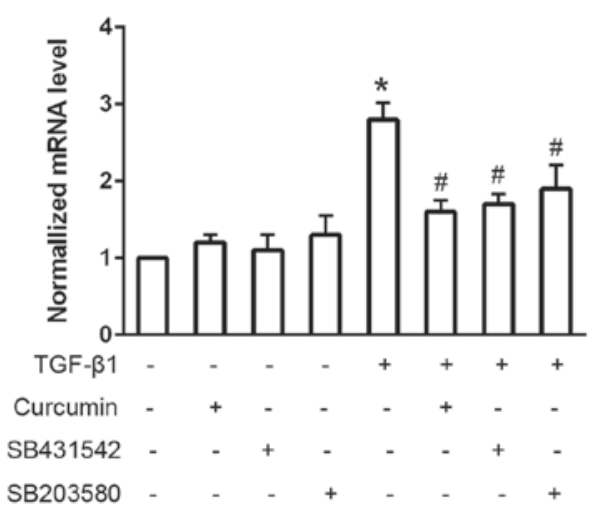

B

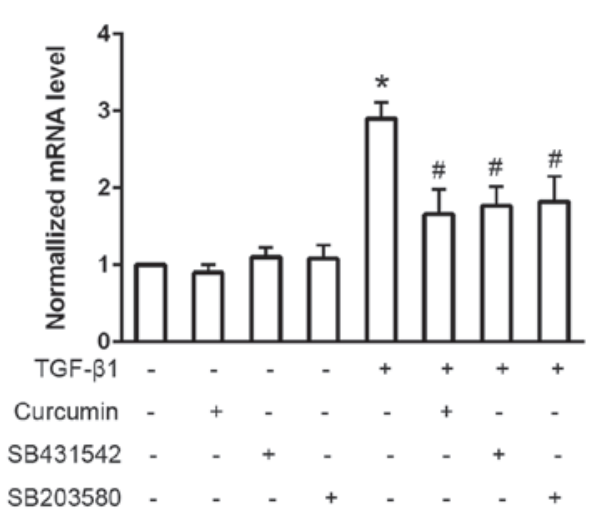

Figure 4. Smad2 and p38 inhibitors, SB431542 and SB20380 respectively, suppressed (A) $\alpha$-smooth muscle actin (SMA) and (B) collagen I (ColI) expression levels. Cardiac fibroblasts (CFs) were treated with $10 \mathrm{ng} / \mathrm{ml}$ transforming growth factor (TGF)- $\beta 1$ for $24 \mathrm{~h}$ in the presence or absence of $10 \mu \mathrm{M} \mathrm{SB} 431542$ or SB20380 inhibitors. mRNA expression of the control CFs was normalized to 1 -fold. ${ }^{*} \mathrm{P}<0.05$ vs. control group; ${ }^{*} \mathrm{P}<0.05$ vs. TGF- $\beta 1$ group.

\section{Results}

Treatment with curcumin impairs TGF-ß1-induced cardiac fibroblast differentiation. To investigate the effects of curcumin on the differentiation of CFs into myofibroblasts, western blotting was used to detect the protein expression levels of $\alpha$-SMA and ColI (Fig. 1A). The results demonstrated that curcumin significantly suppressed the TGF- $\beta 1$-induced protein expression of $\alpha$-SMA and ColI in CFs $(\mathrm{P}<0.05)$, in a dose-dependent manner. These anti-differentiation effects were further validated by immunofluorescence staining of $\alpha$-SMA (Fig. 1B). As compared with untreated CFs, cells induced by $10 \mathrm{ng} / \mathrm{ml}$ TGF- $\beta 1$ exhibited bright fluorescence staining signals for $\alpha$-SMA and prominent stress fibers; by contrast, treatment with $20 \mu \mathrm{M}$ curcumin significantly attenuated $\alpha$-SMA fluorescence signals and morphological characteristics of $\mathrm{CFs}$ induced by $10 \mathrm{ng} / \mathrm{ml}$ TGF- $\beta 1(\mathrm{P}<0.05)$. Furthermore, RT-qPCR was performed to analyze the
mRNA expression levels of $\alpha$-SMA and ColI. The results demonstrated that $\alpha$-SMA and ColI mRNA expression levels in TGF- $\beta 1$-induced CFs were also significantly and dose-dependently decreased following treatment with curcumin $(\mathrm{P}<0.05$; Fig. 2).

TGF- $\beta 1$-activated Smad 2 and p38 signaling pathways in $C F$ s. To investigate the downstream signaling pathways in TGF- $\beta 1$-stimulated CFs, the levels of activated (phosphorylated) Smad2 and p38 were measured at various time points between 15 and 60 min after TGF- $\beta 1$ administration (Fig. 3). As determined by western blotting, phosphorylated Smad 2 and p38 (p-Smad2 and p-p38) levels were significantly increased following stimulation with TGF- $\beta 1(\mathrm{P}<0.05)$. Total Smad2 and p38 protein expression levels were also examined and found to be unaffected by TGF- $\beta 1$ administration. These results indicate that treatment with TGF- $\beta 1$ activates the Smad2 and p38 signaling pathways in CFs. 

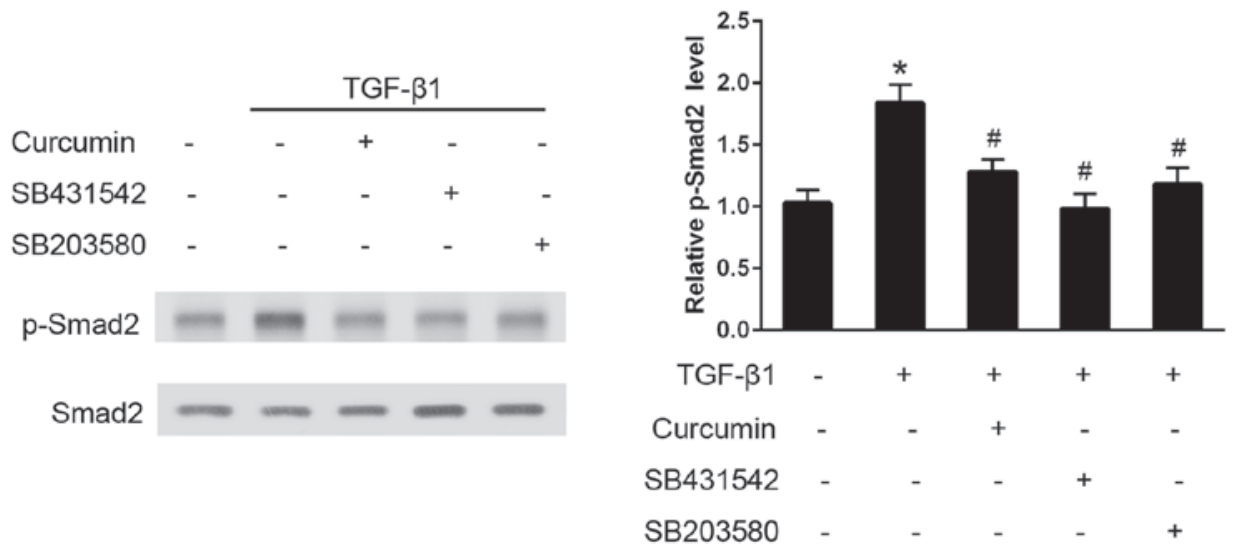

Figure 5. Curcumin administration inhibited the transforming growth factor (TGF)- $\beta 1$-induced activation of Smad2 in cardiac fibroblasts (CFs). CFs were pretreated with $20 \mu \mathrm{mol} / 1$ curcumin, $10 \mu \mathrm{M} \mathrm{SB} 431542$ (Smad2 inhibitor) or $10 \mu \mathrm{M} \mathrm{SB} 203580$ (p38 inhibitor) for 30 min, followed by treatment with $10 \mathrm{ng} / \mathrm{ml} \mathrm{TGF}-\beta 1$ for $30 \mathrm{~min}$. Densitometric analysis was performed to quantify the phosphorylation levels, which are presented as the ratio between the optical densities of the $\mathrm{p}-\mathrm{Smad} 2$ and total Smad2 bands. ${ }^{*} \mathrm{P}<0.05$ vs. the control group; ${ }^{*} \mathrm{P}<0.05$ vs. the TGF- $\beta 1$ group.
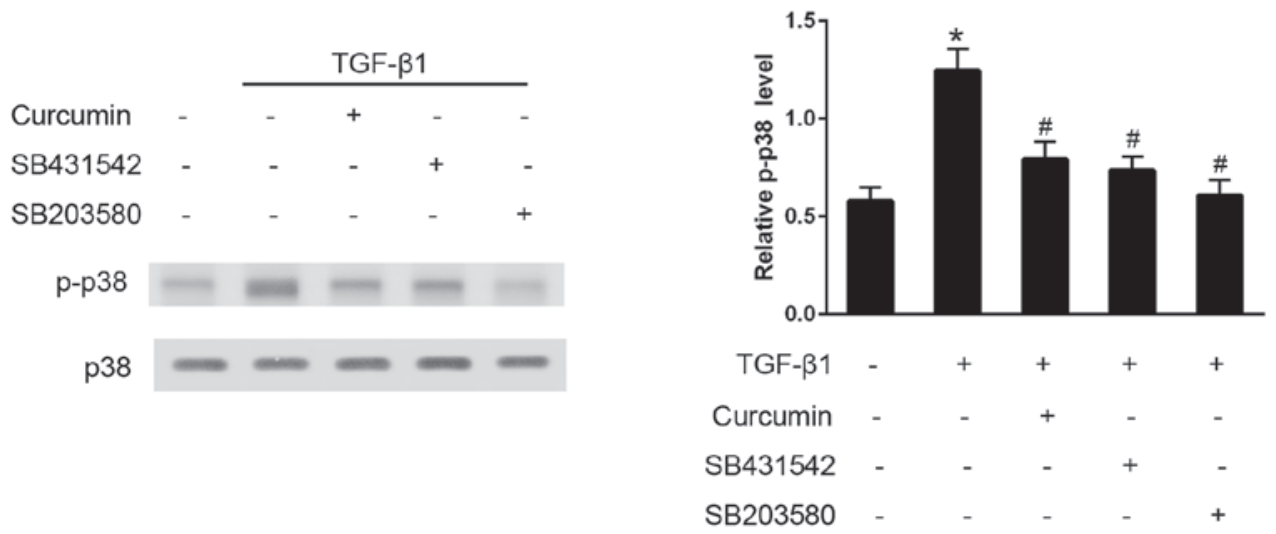

Figure 6. Curcumin administration inhibited the transforming growth factor (TGF)- $\beta 1$-induced activation of $\mathrm{p} 38$ in cardiac fibroblasts (CFs). CFs were pretreated with $20 \mu \mathrm{mol} / 1$ curcumin, $10 \mu \mathrm{M} \mathrm{SB} 431542$ (Smad2 inhibitor) or $10 \mu \mathrm{M} \mathrm{SB} 203580$ (p38 inhibitor) for 30 min, followed by treatment with $10 \mathrm{ng} / \mathrm{ml}$ TGF- $\beta 1$ for $30 \mathrm{~min}$. Densitometric analysis was performed to quantify the phosphorylation levels, which are presented as the ratio between the optical densities of the p-p38 and total $\mathrm{p} 38$ bands. ${ }^{*} \mathrm{P}<0.05$ vs. control group; ${ }^{*} \mathrm{P}<0.05$ vs. TGF- $\beta 1$ group.

Smad2 inhibitor, SB431542, and p38 inhibitor, SB203580, suppress TGF- $\beta 1$-induced $\alpha$-SMA and Coll expression levels. To assess whether Smad2 and $\mathrm{p} 38$ were associated with the TGF- $\beta 1$-induced differentiation of CFs, the Smad2 and p38 inhibitors, SB431542 and SB203580, respectively, were used to block these signaling pathways (Fig. 4). When administered alone, SB431542 and SB203580 exhibited no significant effect on cardiac fibroblast differentiation, as detected by consistent mRNA $\alpha$-SMA and ColI expression levels $(\mathrm{P}>0.05)$. However, pretreatment with SB431542 or SB203580 prior to TGF- $\beta 1$ administration significantly suppressed the TGF- $\beta 1$-induced mRNA expression levels of $\alpha$-SMA and ColI $(\mathrm{P}<0.05)$.

Curcumin inhibits TGF- $\beta 1$-induced activation of the Smad2 signaling pathway. In CFs, TGF- $\beta 1$ administration induced an early and significant increase in p-Smad 2 expression levels over the baseline value in $30 \mathrm{~min}$, as analyzed using western blotting $(\mathrm{P}<0.05$; Fig. 5). Pre-treatment with curcumin induced a significant decrease in the degree of Smad2 phosphorylation and, as hypothesized, SB431542 $(\mathrm{P}<0.05)$, which is a well established TGF- $\beta$ R-Smad2 inhibitor, effectively eliminated the activation of Smad2. Furthermore, pre-treatment with the p38 inhibitor, SB203580, also significantly attenuated the TGF- $\beta 1$-induced Smad 2 phosphorylation $(\mathrm{P}<0.05)$.

Curcumin inhibits TGF- $\beta 1$-induced activation of the $p 38$ pathway. The effects of curcumin on the TGF- $\beta 1$-induced activation of the p38 signaling pathway were also examined. Western blot analysis demonstrated that, in TGF- $\beta 1$-stimulated $\mathrm{CFs}$, curcumin pre-treatment significantly decreased p-p38 expression levels, and SB203580 effectively eliminated p38 activation $(\mathrm{P}<0.05)$. Furthermore, SB431542 administration also significantly inhibited the phosphorylation of $\mathrm{p} 38$, thus preventing its activation $(\mathrm{P}<0.05$; Fig. 6$)$.

\section{Discussion}

In the present study, curcumin was demonstrated to dose-dependently inhibit the TGF- $\beta 1$-induced cardiac fibroblast differentiation. Furthermore, the results indicated that these effects may be mediated by the Smad2 and p 38 signaling pathways. Therefore, the present results suggested 
that curcumin may be a potential therapeutic agent for the treatment of myocardial fibrosis, which has been associated with the pathogenesis of heart failure (1).

The transformation of CFs to cardiac myofibroblasts is a key event in cardiac fibrosis. Cardiac myofibroblasts are absent from normal myocardium, however, they are the predominant source of excessive extracellular collagen in cardiac fibrosis $(20,21)$. In addition to aggravating cardiac dysfunction, previous studies have demonstrated that the persistence of cardiac myofibroblasts also contributes to malignant arrhythmia (22-24). Therefore, factors associated with the formation of cardiac myofibroblasts are of considerable clinical interest.

High expression levels of $\alpha$-SMA are a hallmark of the formation of cardiac myofibroblasts $(24,25)$. Collagen, particularly fibrillar ColI, is the predominant component of the ECM and is excessively synthesized by cardiac myofibroblasts $(26,27)$. The results of the present study demonstrated that curcumin administration effectively suppressed TGF- $\beta 1$-induced cardiac fibroblast differentiation, as determined by the decreased expression of $\alpha$-SMA and ColI, at the protein and mRNA levels. These findings are consistent with previous studies which have demonstrated that curcumin is capable of inducing anti-fibrotic effects in cultured CFs $(10,28)$.

TGF- $\beta 1$, which is the most well-characterized cytokine, induces the differentiation of CFs to cardiac myofibroblasts (29,30). Activation of the Smad cascade has an essential role in the differentiation of myofibroblasts $(15,16,31)$ and is regarded as the classical mediator of TGF- $\beta 1$. Following TGF- $\beta 1$ stimulation, the TGF- $\beta 1 \mathrm{RI}$ serine-threonine kinase phosphorylates the receptor-Smads (R-Smads), and Smad2 subsequently forms a complex with Smad3 that, in turn, associates with a Co-Smad $(\operatorname{Smad} 4)$ and translocates into the nucleus, where it acts as a transcription factor (32). In addition to Smad-dependent pathways, previous studies have suggested that Smad independent pathways, such as p38 MAPK, may also play a pivotal role in TGF- $\beta 1$-mediated differentiation $(17,33)$. In the present study, inhibition of Smad 2 and p38 consistently attenuated the TGF- $\beta 1$-induced cardiac fibroblast differentiation, whereas curcumin was able to reduce the activation of Smad2 and p38 molecular signaling. Therefore, curcumin may have inhibited TGF- $\beta 1$-induced activation via the Smad2 and p38 signaling pathways. Furthermore, the Smad2 inhibitor SB431542 effectively prohibited the phosphorylation of $\mathrm{p} 38$, and the $\mathrm{p} 38$ inhibitor SB203580 reduced the phosphorylation of Smad2. These results demonstrated that there is cross-talk or interaction between these two signaling pathways; however, the detailed mechanism and biological consequences of Smad2 and p38 activation remain unclear. This potential interaction among Smad2, p38 and other associated pathways requires further investigation.

In conclusion, the results of the present study suggested that curcumin has fibrosis suppressor properties and an inhibitory effect on TGF- $\beta 1$-induced cardiac fibroblast differentiation, which may be mediated via the suppression of the Smad2 and p38 signaling pathways. Therefore, curcumin may be a potential novel therapeutic agent for the treatment of cardiac fibrosis.

\section{References}

1. Daskalopoulos EP, Janssen BJ and Blankesteijn WM: Myofibroblasts in the infarct area: Concepts and challenges. Microsc Microanal 18: 35-49, 2012.

2. Gupta SC, Patchva S, Koh W and Aggarwal BB: Discovery of curcumin, a component of golden spice and its miraculous biological activities. Clin Exp Pharmacol Physiol 39: 283-299, 2012.

3. Chiu J, Khan ZA, Farhangkhoee H and Chakrabarti S: Curcumin prevents diabetes-associated abnormalities in the kidneys by inhibiting p300 and nuclear factor- $\kappa$ B. Nutrition 25: 964-972, 2009.

4. Kolodziejczyk J, Olas B, Saluk-Juszczak J and Wachowicz B: Antioxidative properties of curcumin in the protection of blood platelets against oxidative stress in vitro. Platelets 22: 270-276, 2011.

5. Abe Y, Hashimoto S and Horie T: Curcumin inhibition of inflammatory cytokine production by human peripheral blood monocytes and alveolar macrophages. Pharmacol Res 39: 41-47, 1999.

6. Teiten MH, Gaascht F, Cronauer M, Henry E, Dicato M and Diederich M: Anti-proliferative potential of curcumin in androgen-dependent prostate cancer cells occurs through modulation of the Wingless signaling pathway. Int J Oncol 38: 603-611, 2011.

7. Hartojo W, Silvers AL, Thomas DG, Seder CW, Lin L, Rao H, Wang Z, Greenson JK, Giordano TJ, Orringer MB, et al: Curcumin promotes apoptosis, increases chemosensitivity, and inhibits nuclear factor kappaB in esophageal adenocarcinoma. Transl Oncol 3: 99-108, 2010

8. Chanvorachote P, Pongrakhananon V, Wannachaiyasit S, Luanpitpong S, Rojanasakul Y and Nimmannit U: Curcumin sensitizes lung cancer cells to cisplatin-induced apoptosis through superoxide anion-mediated Bcl-2 degradation. Cancer Invest 27: 624-635, 2009.

9. Ghosh SS, Salloum FN, Abbate A, Krieg R, Sica DA, Gehr TW and Kukreja RC: Curcumin prevents cardiac remodeling secondary to chronic renal failure through deactivation of hypertrophic signaling in rats. Am J Physiol Heart Circ Physiol 299: H975-H984, 2010.

10. Li HL, Liu C, de Couto G, Ouzounian M, Sun M, Wang AB, Huang Y, He CW, Shi Y, Chen X, et al: Curcumin prevents and reverses murine cardiac hypertrophy. J Clin Invest 118: 879-893, 2008.

11. Wang NP, Wang ZF, Tootle S, Philip T and Zhao ZQ: Curcumin promotes cardiac repair and ameliorates cardiac dysfunction following myocardial infarction. Br J Pharmacol 167: 1550-1562, 2012.

12. Morimoto T, Sunagawa Y, Kawamura T, Takaya T, Wada H, Nagasawa A, Komeda M, Fujita M, Shimatsu A, Kita T and Hasegawa K: The dietary compound curcumin inhibits p300 histone acetyltransferase activity and prevents heart failure in rats. J Clin Invest 118: 868-878, 2008.

13. Soetikno V, Sari FR, Sukumaran V, Lakshmanan AP, Mito S, Harima M, Thandavarayan RA, Suzuki K, Nagata M, Takagi R and Watanabe K: Curcumin prevents diabetic cardiomyopathy in streptozotocin-induced diabetic rats: Possible involvement of PKC-MAPK signaling pathway. Eur J Pharm Sci 47: 604-614, 2012.

14. Biernacka A, Dobaczewski M and Frangogiannis NG: TGF- $\beta$ signaling in fibrosis. Growth factors 29: 196-202, 2011.

15. Cucoranu I, Clempus R, Dikalova A, Phelan PJ, Ariyan S, Dikalov $\mathrm{S}$ and Sorescu D: NAD (P) H oxidase 4 mediates transforming growth factor- $\beta 1$-induced differentiation of cardiac fibroblasts into myofibroblasts. Circ Res 97: 900-907, 2005.

16. Dobaczewski M, Bujak M, Li N, Gonzalez-Quesada C, Mendoza LH, Wang XF and Frangogiannis NG: Smad3 signaling critically regulates fibroblast phenotype and function in healing myocardial infarction. Circ Res 107: 418-428, 2010.

17. Deaton RA, Su C, Valencia TG and Grant SR: Transforming growth factor- $\beta 1$-induced expression of smooth muscle marker genes involves activation of PKN and p38 MAPK. J Biol Chem 280: 31172-31181, 2005.

18. Yan-Hong F, Hui D, Qing P, Lei S, Hai-Chang W, Wei Z and Yan-Jie C: Effects of arginine vasopressin on differentiation of cardiac fibroblasts into myofibroblasts. J Cardiovasc Pharmacol 55: 489-495, 2010.

19. Livak KJ and Schmittgen TD: Analysis of relative gene expression data using real-time quantitative PCR and the 2(-Delta Delta C(T)) Method. Methods 25: 402-408, 2001.

20. Porter KE and Turner NA: Cardiac fibroblasts: at the heart of myocardial remodeling. Pharmacol Ther 123: 255-278, 2009. 
21. van den Borne SW, Diez J, Blankesteijn WM, Verjans J, Hofstra L and Narula J: Myocardial remodeling after infarction: the role of myofibroblasts. Nat Rev Cardiol 7: 30-37, 2010.

22. Sabbah HN, Sharov VG, Lesch M and Goldstein S: Progression of heart failure: A role for interstitial fibrosis. Mol Cell Biochem 147: 29-34, 1995.

23. McDowell KS, Arevalo HJ, Maleckar MM and Trayanova NA: Susceptibility to arrhythmia in the infarcted heart depends on myofibroblast density. Biophys J 101: 1307-1315, 2011.

24. Rohr S: Myofibroblasts in diseased hearts: new players in cardiac arrhythmias? Heart Rhythm 6: 848-856, 2009.

25. Leask A: Potential therapeutic targets for cardiac fibrosis TGF $\beta$, angiotensin, endothelin, CCN2 and PDGF, partners in fibroblast activation. Circ Res 106: 1675-1680, 2010.

26. Chapman D, Weber KT and Eghbali M: Regulation of fibrillar collagen types I and III and basement membrane type IV collagen gene expression in pressure overloaded rat myocardium. Circ Res 67: 787-794, 1990.

27. Cleutjens JP, Verluyten MJ, Smiths JF and Daemen MJ: Collagen remodeling after myocardial infarction in the rat heart. Am J Pathol 147: 325-338, 1995.
28. Meng Z, Yu Xh, Chen J, Li L and Li S: Curcumin attenuates cardiac fibrosis in spontaneously hypertensive rats through PPAR- $\gamma$ activation. Acta Pharmacol Sin 35: 1247-1256, 2014.

29. Bujak M and Frangogiannis NG: The role of TGF- $\beta$ signaling in myocardial infarction and cardiac remodeling. Cardiovasc Res 74: 184-195, 2007.

30. Leask A: TGFbeta, cardiac fibroblasts and the fibrotic response. Cardiovasc Res 74: 207-212, 2007.

31. Carthy JM, Garmaroudi FS, Luo Z and McManus BM: Wnt3a induces myofibroblast differentiation by upregulating TGF- $\beta$ signaling through SMAD2 in a $\beta$-catenin-dependent manner. PloS one 6: e19809, 2011

32. Derynck $R$ and Zhang YE: Smad-dependent and Smad-independent pathways in TGF-beta family signalling. Nature 425: 577-584, 2003.

33. Horowitz JC, Rogers DS, Sharma V, Vittal R, White ES, Cui Z and Thannickal VJ: Combinatorial activation of FAK and AKT by transforming growth factor-beta1 confers an anoikis-resistant phenotype to myofibroblasts. Cell Signal 19: 761-771, 2007. 